\title{
ON EQUIVALENCE OF ANALYTIC FUNCTIONS TO RATIONAL REGULAR FUNCTIONS
}

\author{
WOJCIECH KUCHARZ
}

(Received 15 April 1986)

Communicated by J. H. Rubinstein

\begin{abstract}
We give sufficient conditions for an analytic function from $\mathbf{R}^{n}$ to $\mathbf{R}$ to be analytically equivalent to a rational regular function.
\end{abstract}

1980 Mathematics subject classification (Amer. Math. Soc.): 57 R 45, 58 C 27, 32 C 40.

\section{Introduction}

We say that two functions $f_{1}, f_{2}: \mathbf{R}^{n} \rightarrow \mathbf{R}$ are analytically equivalent if $f_{2}=f_{1} \circ \boldsymbol{\sigma}$ for some analytic diffeomorphism $\sigma: \mathbf{R}^{n} \rightarrow \mathbf{R}^{n}$.

A function $\varphi: \mathbf{R}^{n} \rightarrow \mathbf{R}$ is said to be rational regular or, simply, regular if it can be written as $\varphi=\lambda / \mu$, where $\lambda$ and $\mu$ are polynomial functions on $\mathbf{R}^{n}$ and $\mu$ does not vanish on $\mathbf{R}^{n}$.

In this paper we study the following problem.

Problem 1.1. Under what conditions is a given analytic function $f: \mathbf{R}^{n} \rightarrow \mathbf{R}$ analytically equivalent to a regular function?

Some variations of this problem have been investigated in [1], [2], [5] and [6]. It was Thom's paper [6], which gave an impulse for research in this direction.

First let us observe that if $f$ is analytically equivalent to a regular function, then for each point $x$ in $\mathbf{R}^{n}$ the germ $f_{x}$ of $f$ at $x$ is locally analytically equivalent to a germ of a regular function, that is, $f_{x} \circ \sigma_{x}$ is a germ of a regular function for

(C) 1987 Australian Mathematical Society $0263-6115 / 87 \$ A 2.00+0.00$ 
some local analytic diffeomorphism $\sigma_{x}:\left(\mathbf{R}^{n}, x\right) \rightarrow\left(\mathbf{R}^{n}, x\right)$. The following example shows that even the nicest local behavior of $f$ does not guarantee analytic equivalence of $f$ to a regular function.

EXAMPLE 1.2. Let $f: \mathbf{R} \rightarrow \mathbf{R}$ be an analytic function with no critical point which has two distinct horizontal asymptotes, for example, $f(x)=\arctan x$. Clearly, for each point $x$ in $\mathbf{R}$, the gerem $f_{x}$ is locally analytically equivalent to the germ of the identity. However, $f$ is not analytically equivalent to a regular function.

The only obstruction which prevents the function $f$ of Example 1.2 from being analytically equivalent to a regular function is its "bad" behavior at "infinity" (cf. Theorem 1.5). To avoid this, we impose some restrictions on analytic functions under consideration.

Let $S^{n}$ be the unit $n$-dimensional sphere and let $a=(0, \ldots, 0,1) \in S^{n}$.

DEFINITION 1.3. An analytic function $f: \mathbf{R}^{n} \rightarrow \mathbf{R}$ is said to be meromorphic at infinity if there exists an analytic diffeomorphism $\tau: S^{n} \backslash\{a\} \rightarrow \mathbf{R}^{n}$ such that $f \circ \tau$ extends to a meromorphism function on $S^{n}$, that is, there exist a connected neighborhood $U$ of $a$ in $S^{n}$ and analytic functions $u, v: U \rightarrow \mathbf{R}$ such that $v$ is not identically equal to 0 on $U$ and $f \circ \tau=u / v$ on $U \backslash v^{-1}(0)$ (it is well-known that $u$ and $v$ can be selected with $\left.v^{-1}(0)=\{a\}\right)$.

Definition 1.3 is quite natural in the context of this paper. Indeed, we have

Proposition 1.4. If an analytic function $f: \mathbf{R}^{n} \rightarrow \mathbf{R}$ is analytically equivalent to a regular function, thenit is meromorphic at infinity.

Proof. Choose an analytic diffeomorphism $\sigma: \mathbf{R}^{n} \rightarrow \mathbf{R}^{n}$ such that $f \circ \sigma$ is a regular function. Let $\rho: S^{n} \backslash\{a\} \rightarrow \mathbf{R}^{n}$ be the stereographic projection from $a$,

$$
\rho\left(x_{1}, \ldots, x_{n}, x_{n+1}\right)=\left(\frac{x_{1}}{1-x_{n+1}}, \ldots, \frac{x_{n}}{1-x_{n+1}}\right) .
$$

Clearly, $f \circ \sigma \circ \rho$ can be written as $f \circ \sigma \circ \rho=\lambda / \mu$, where $\lambda$ and $\mu$ are polynomial functions on $\mathbf{R}^{n+1}$ with $\mu$ nonvanishing on $S^{n} \backslash\{a\}$. It follows that $f$ is meromorphic at infinity.

Summarizing, every analytic function from $\mathbf{R}^{n}$ to $\mathbf{R}$, analytically equivalent to a regular function, is locally analytically equivalent to a germ of a regular function and is meromorphic at infinity. It is an interesting question to what extent the converse is true.

Before we formulate our main result, we need to recall a few concepts. Given a point $x$ in $\mathbf{R}^{n}$, we denote by $\mathcal{O}_{x}$ the ring of all analytic function-germs $\left(\mathbf{R}^{n}, 0\right) \rightarrow \mathbf{R}$. If $f_{x}$ belongs to $\mathcal{O}_{x}$, then $\Delta\left(f_{x}\right)$ denotes the ideal of $\mathcal{O}_{x}$ generated by the first 
partial derivatives of $f_{x}$. The Milnor number of $f_{x}$ is the dimension of the R-vector space $\mathcal{O}_{x} / \Delta\left(f_{x}\right)$. It is well-known that if the Milnor number of $f_{x}$ is finite, then given any analytic germ $g_{x}$ in $\mathcal{O}_{x}$ with $g_{x}-f_{x}$ being $k$-flat at $x$, one can find a local analytic diffeomorphism $\sigma_{x}:\left(\mathbf{R}^{n}, x\right) \rightarrow\left(\mathbf{R}^{n}, x\right)$ satisfying $g_{x}=$ $f_{x} \circ \sigma_{x}$, provided that $k$ is sufficiently large [7]. In particular, $f_{x}$ is locally analytically equivalent to the germ at $x$ of a polynomial function.

THEOREM 1.5. Let $f: \mathbf{R}^{n} \rightarrow \mathbf{R}$ be an analytic function whose germ at each point in $\mathbf{R}^{n}$ has a finite Milnor number. Then $f$ is analytically equivalent to a regular function if and only if the set of critical poins of $f$ is finite and $f$ is meromorphic at infinity.

We conclude this section by recalling that the set of all analytic functions $f: \mathbf{R}^{n} \rightarrow \mathbf{R}$ such that for each point $x$ in $\mathbf{R}^{n}$ the germ $f_{x}$ has a finite Milnor number is very large [7].

\section{Proof of Theorem 1.5}

Let $M$ be a $C^{\infty}$ manifold. Denote by $\mathscr{E}(M)$ the ring of $C^{\infty}$ functions on $M$. Let $X_{1}, \ldots, X_{n}$ be $C^{\infty}$ vector fields on $M$ generating the $\mathscr{E}(M)$-module of all $C^{\infty}$ vector fields on $M$. Given an element $\left(f_{1}, f_{2}\right)$ in $\mathscr{E}(M)^{2}$, we define $I\left(f_{1}, f_{2}\right)$ to be the ideal of $\mathscr{E}(M)$ generated by all $2 \times 2$ minors of the matrix

$$
\left(\begin{array}{llll}
f_{1} & X_{1} f_{1} & \cdots & X_{n} f_{1} \\
f_{2} & X_{1} f_{1} & \cdots & X_{n} f_{2}
\end{array}\right)
$$

and $S\left(f_{1}, f_{2}\right)$ to be the set of zeros of $I\left(f_{1}, f_{2}\right)$.

We shall need the following two auxiliary results.

LeMma 2.1. Let $M$ be a compact $C^{\infty}$ manifold. Let $\left(f_{1}, f_{2}\right)$ be an element in $\mathscr{E}(M)^{2}$ and let $Z$ be a subset of $M$. Assume that the ideal $I\left(f_{1}, f_{2}\right)^{2}$ is closed in the $C^{\infty}$ topology and the ideal $I(Z)$ of all functions in $\mathscr{E}(M)$ vanishing on $Z$ is finitely generated. Then there exists a neighborhood $\mathscr{V}$ of 0 in $\mathscr{E}(M)$ such that for every element $\left(g_{1}, g_{2}\right)$ in $\mathscr{E}(M)^{2}$ with

$$
g_{i}-f_{i} \in I(Z) I\left(f_{1}, f_{2}\right)^{2} \cap \mathscr{V}, \quad i=1,2,
$$

one can find a $C^{\infty}$ diffeomorphism $\sigma: M \rightarrow M$ and an element $u$ in $\mathscr{E}(M)$ satisfying $\sigma(x)=x$ for all $x$ in $Z, u>0$ and $\left(g_{1}, g_{2}\right)=\left(u\left(f_{1} \circ \sigma\right), u\left(f_{2} \circ \sigma\right)\right)$.

Proof. If $Z$ is the empty set, then Lemma 2.1 is a particular case of [2], Theorem 2.1 with, in the notation of [2], $p=2$ and $G$ being the subgroup of $G l(2, \mathbf{R})$ of all diagonal matrices having the diagonal of the form $(\lambda, \lambda)$, where 
$\lambda>0$. In the general case only an obvious modification of the proof of [2], Theorem 2.1 is necessary.

LemMA 2.2. Let $M$ be a compact real analytic manifold. Let $\left(f_{1}, f_{2}\right)$ be an element of $\mathscr{E}(M)^{2}$. Assume that the set $S\left(f_{1}, f_{2}\right)$ is discrete and $f_{i}$ is analytic in a neighborhood of $S\left(f_{1}, f_{2}\right)$ for $i=1,2$. Then there exist a neighborhood $\mathscr{V}$ of 0 in $\mathscr{E}(M)$ and a positive integer $k$ such that for each element $\left(g_{1}, g_{2}\right)$ in $\mathscr{E}(M)^{2}$ with $g_{i}-f_{i}$ belonging to $\mathscr{V}$ and being $k$-flat at $S\left(f_{1}, f_{2}\right), i=1,2$, one has $S\left(g_{1}, g_{2}\right)=$ $S\left(f_{1}, f_{2}\right)$.

Proof. Since one can choose finitely many analytic vector fields on $M$ generating the $\mathscr{E}(M)$-module of all $C^{\infty}$ vector fields on $M$, the ideal $I\left(f_{1}, f_{2}\right)$ is generated by finitely many $C^{\infty}$ functions which are analytic in a neighborhood of $S\left(f_{1}, f_{2}\right)$. Now the conclusion is a consequence of the following observation.

Let $u: \mathbf{R}^{n} \rightarrow \mathbf{R}$ be an analytic function with $u^{-1}(0)=\{0\}$. By the Lojasiewicz inequality [7], there exist positive real numbers $c$ and $\varepsilon$ and a positive integer $l$ such that

$$
|u(x)| \geqslant c\|x\|^{l} \text { for }\|x\| \leqslant \varepsilon .
$$

If $v: \mathbf{R}^{n} \rightarrow \mathbf{R}$ is a $C^{\infty}$ function such that $v-u$ is $l$-flat at 0 in $\mathbf{R}^{n}$, then, by the Taylor formula,

$$
\begin{aligned}
|v(x)| & \geqslant|u(x)|-|v(x)-u(x)| \\
& \geqslant c\|x\|^{l}-\left(\sup \left\{\sum_{\alpha} \frac{1}{\alpha !}\left|D^{\alpha}(v-u)(x)\right|:\|x\| \leqslant \varepsilon\right\}\right)\|x\|^{l+1} .
\end{aligned}
$$

We shall also need a special local version of Lemma 2.1. Let $\left(f_{1}, f_{2}\right):\left(\mathbf{R}^{n}, 0\right) \rightarrow$ $\mathbf{R}^{2}$ be a $C^{\infty}$ map-germ. Denote by $I_{0}\left(f_{1}, f_{2}\right)$ the ideal of the ring $\mathscr{E}_{0}$ of $C^{\infty}$ function-germs $\left(\mathbf{R}^{n}, 0\right) \rightarrow \mathbf{R}$ generated by all $2 \times 2$ minors of the matrix

$$
M_{0}\left(f_{1}, f_{2}\right)=\left(\begin{array}{llll}
f_{1} & \frac{\partial f_{1}}{\partial x_{1}} & \cdots & \frac{\partial f_{1}}{\partial x_{n}} \\
f_{2} & \frac{\partial f_{2}}{\partial x_{1}} & \cdots & \frac{\partial f_{2}}{\partial x_{n}}
\end{array}\right) .
$$

LEMMA 2.3. Let $\left(f_{1}, f_{2}\right):\left(\mathbf{R}^{n}, 0\right) \rightarrow \mathbf{R}^{2}$ be an analytic map-germ. Assume that the set-germ of zeros of $I_{0}\left(f_{1}, f_{2}\right)$ is contained in $\{0\}$. Then there exists a positive integer $k$ such that for each analytic map-germ $\left(g_{1}, g_{2}\right):\left(\mathbf{R}^{n},\right) \rightarrow \mathbf{R}^{2}$ with $g_{i}-f_{i}$ being $k$-flat at 0 in $\mathbf{R}^{n}, i=1,2$, one can find a local $C^{1}$ orientation preserving diffeomorphism $\sigma:\left(\mathbf{R}^{n}, 0\right) \rightarrow\left(\mathbf{R}^{n}, 0\right)$ and a $C^{1}$ function-germ $u:\left(\mathbf{R}^{n}, 0\right) \rightarrow \mathbf{R}$, both analytic off the origin and satisfying $u>0$ and $\left(g_{1}, g_{2}\right)=\left(u\left(f_{1} \circ \sigma\right), u\left(f_{2} \circ \sigma\right)\right)$. 
Proof. Let $k$ be a positive integer and let $\left(g_{1}, g_{2}\right): \mathbf{R}^{n} \rightarrow \mathbf{R}^{2}$ be an analytic map-germ such that $g_{i}-f_{i}$ is $k$-flat at 0 in $\mathbf{R}^{n}$ for $i=1,2$. Define $F(x, t)=$ $\left(F_{1}(x, t), F_{2}(x, t)\right)$ by $F_{i}(x, t)=(1-t) f_{i}(x)+\operatorname{tg}_{i}(x)$ for $t$ in $[0,1]$ and $i=1,2$. Let $\mathcal{O}_{n}[0,1]$ be the ring of analytic function-germs $\left(\mathbf{R}^{n} \times \mathbf{R},\{0\} \times[0,1]\right) \rightarrow \mathbf{R}$. Set

$$
M_{0}\left(F_{1}, F_{2}\right)=\left(\begin{array}{llll}
F_{1} & \frac{\partial F_{1}}{\partial x_{1}} \cdots & \frac{\partial F_{1}}{\partial x_{n}} \\
F_{2} & \frac{\partial F_{2}}{\partial x_{1}} \cdots & \frac{\partial F_{2}}{\partial x_{n}}
\end{array}\right)
$$

and

$$
e_{1}=\left(\begin{array}{l}
1 \\
0
\end{array}\right), \quad e_{2}=\left(\begin{array}{l}
0 \\
1
\end{array}\right) \text {. }
$$

If $\Delta$ is a $2 \times 2$ minor of $M_{0}\left(F_{1}, F_{2}\right)$, then $\Delta e_{i}$ is a linear combination of the columns of $M_{0}\left(F_{1}, F_{2}\right)$ with coefficients in $\mathcal{O}_{n}[0,1]$. Let $\delta$ be the sum of squares of all $2 \times 2$ minors of $M_{0}\left(F_{1}, F_{2}\right)$. Then

$$
-\delta(x, t) \frac{\partial F}{\partial t}(x, t)=\sum_{j=1}^{n} \xi_{j}(x, t) \frac{\partial F}{\partial x_{j}}(x, t)+\eta(x, t) F(x, t)
$$

for some $\xi_{j}, \eta$ in $\mathcal{O}_{n}[0,1]$. Moreover, $\xi_{j}(\cdot, t)$ and $\eta(\cdot, t)$ are $k$-flat at 0 in $\mathbf{R}^{n}$ for all $t$ in $[0,1]$. Notice that $\delta(\cdot, 0)$ is the sum of squares of all $2 \times 2$ minors of the matrix $M_{0}\left(f_{1}, f_{2}\right)$. Thus the set-germ of zeros of $\delta(\cdot, 0)$ is contained in $\{0\}$. By the Lojasiewicz inequality [7],

$$
\delta(x, 0) \geqslant c\|x\|^{l} \text { for }\|x\|<\boldsymbol{\alpha},
$$

where $c, \alpha>0$ and $l$ is a positive integer. Now assume that $k$ has been chosen satisfying $k \geqslant 2(l+1)$. Since $\delta(\cdot, t)-\delta(\cdot, 0)$ is $(k-1)$-flat at 0 in $\mathbf{R}^{n}$, it follows that

$$
\delta(x, t) \geqslant \frac{c}{2}\|x\|^{l} \quad \text { for }\|x\|<\beta \quad \text { and } t \in[0,1],
$$

where $\beta>0$. By choice of $k$, for each $j=1, \ldots, n, \xi_{j} / \delta$ and $\eta / \delta$ have $C^{1}$ extensions $\xi_{j}^{\prime}$ and $\eta^{\prime}$ on a neighborhood of $\{0\} \times[0,1]$ vanishing on $\{0\} \times[0,1]$. One also has

$$
-\frac{\partial F}{\partial t}(x, t)=d_{2} F(x, t)\left(\xi^{\prime}(x, t)\right)+\eta^{\prime}(x, t) F(x, t),
$$

where

$$
\xi^{\prime}(x, t)=\sum_{j=1}^{n} \xi^{\prime}(x, t) \frac{\partial}{\partial x_{j}} .
$$


Let $\tau:\left(\mathbf{R}^{n} \times \mathbf{R},\{0\} \times[0,1]\right) \rightarrow\left(\mathbf{R}^{n}, 0\right)$ be a $C^{1}$ map-germ satisfying

$$
\left\{\begin{array}{l}
\frac{\partial \tau}{\partial t}(x, t)=\xi^{\prime}(\tau(x, t), t), \\
\tau(x, 0)=x
\end{array}\right.
$$

and let

$$
v(x, t)=\exp \left(-\int_{0}^{t} \eta^{\prime}(\tau(x, s), s) d s\right)
$$

Notice that

$$
\frac{\partial}{\partial t}(F(\tau(x, t), t))=-\eta^{\prime}(\tau(x, t), t) F(\tau(x, t), t)
$$

and

$$
\frac{\partial}{\partial t}(v(x, t) F(x, 0))=-\eta^{\prime}(\tau(x, t), t)(v(x, t) F(x, 0)) .
$$

By the uniqueness of the solution of ordinary differential equations, one obtains $F(\tau(x, t), t)=v(x, t) F(x, 0)$. It suffices to set $\sigma^{-1}(x)=\tau(x, 1)$ and $u(x)=$ $v(\sigma(x), 1)$. Clearly, $\sigma$ and $u$ are analytic off the origin and the conclusion follows.

Let $M$ and $N$ be $C^{\infty}$ manifolds and let $f: M \rightarrow N$ be a $C^{\infty}$ map. The set of critical points of $f$ will be denoted by $\Sigma(f)$ and the germ of $f$ at a point $x$ in $M$ by $f_{x}$. If $X$ is a subset of $\mathbf{R}^{n}$, then by a polynomial function on $X$ we shall mean the restriction to $X$ of a polynomial function on $\mathbf{R}^{n}$.

Proof of Theorem 1.5. It follows from the assumption that the set $\Sigma(f)$ is discrete. Assume that $f \circ \sigma$ is a regular function for some analytic diffeomorphism $\sigma: \mathbf{R}^{n} \rightarrow \mathbf{R}^{n}$. Clearly, the set $\Sigma(f \circ \sigma)$ is semi-algebraic. Since every semialgebraic set has only finitely many connected components [3], the set $\Sigma(f \circ \sigma)$ and, hence also $\Sigma(f)$, is finite. By Proposition 1.4, $f$ is meromorphic at infinity.

Now suppose that $\Sigma(f)$ is finite and $f$ is meromorphic at infinity. Let $\tau: S^{n} \backslash\{a\} \rightarrow \mathbf{R}^{n}$ be an analytic diffeomorphism and let $u_{1}, u_{2}: U \rightarrow \mathbf{R}$ be analytic functions defined on a neighborhood $U$ of $a$ in $S^{n}$ such that $u_{2}$ does not vanish on $U \backslash\{a\}$ and $f \circ \tau=u_{1} / u_{2}$ on $U \backslash\{a\}$. We may assume that $u_{2} \geqslant 0$ on $U$ (replace $u_{1}$ and $u_{2}$ by $u_{1} u_{2}$ and $u_{2}^{2}$, respectively, if necessary). It is easy to construct two $C^{\infty}$ functions $f_{1}, f_{2}: S^{n} \rightarrow \mathbf{R}$ such that $f_{i}=u_{i}$ on a neighborhood of $a, i=1,2, f_{2} \geqslant 0, f_{2}$ does not vanish on $S^{n} \backslash\{a\}$ and $f \circ \tau=f_{1} / f_{2}$ on $S^{n} \backslash\{a\}$. Notice that

$$
S\left(f_{1}, f_{2}\right) \subseteq \sum(f \circ \tau) \cup\{a\} .
$$

Let $\mathscr{V}$ be a small neighborhood of 0 in $\mathscr{E}\left(S^{n}\right)$ and let $k$ be a large positive integer (how small $\mathscr{V}$ is and how large $k$ is will be clear later on). For each $i=1,2$, one can find a polynomial map $\varphi_{i}: S^{n} \rightarrow \mathbf{R}$ such that $f_{i}-\varphi_{i}$ belongs to 
$\mathscr{V}$ and $f_{i}-\varphi_{i}$ is $k$-flat at $\Sigma(f \circ \tau) \cup\{a\}$ (cf. [1], Corollary 1). By Lemma 2.2, $S\left(\varphi_{1}, \varphi_{2}\right)=S\left(f_{1}, f_{2}\right)$. Moreover, for each point $x$ in $\Sigma(f \circ \tau)$ one can find a local $C^{\infty}$ orientation preserving diffeomorphism $\alpha_{x}:\left(S^{n}, x\right) \rightarrow\left(S^{n}, x\right)$ and a $C^{\infty}$ function-germ $v_{x}:\left(S^{n}, x\right) \rightarrow \mathbf{R}$ such that $v_{x}>0$ and $\left(\varphi_{1 x}, \varphi_{2 x}\right)=\left(v_{x}\left(f_{1 x} \circ \alpha_{x}\right)\right.$, $v_{x}\left(f_{2 x} \circ \alpha_{x}\right)$ ) (this follows from the fact that the Milnor number of $f$ at $\tau(x)$ is finite and from [7], p. 169, Theorem 3.11 applied for $p=2$ and the subgroup $G$ of $G l(2, \mathbf{R})$ consisting of all diagonal matrices having the diagonal of the form $(\lambda, \lambda)$, where $\lambda>0)$. By Lemma 2.3, there exists a local $C^{1}$ orientation preserving diffeomorphism $\alpha_{a}:\left(S^{n}, a\right) \rightarrow\left(S^{n}, a\right)$ and a $C^{1}$ function-germ $v_{a}:\left(S^{n}, a\right) \rightarrow \mathbf{R}$ such that $v_{a}>0, \alpha_{a}$ and $v_{a}$ are analytic off $a$ and $\left(\varphi_{1 a}, \varphi_{2 a}\right)=\left(v_{a}\left(f_{1 a} \circ \alpha_{a}\right)\right.$, $\left.v_{a}\left(f_{2 a} \circ \boldsymbol{\alpha}_{a}\right)\right)$. Now one can find a $C^{1}$ diffeomorphism $\beta: S^{n} \rightarrow S^{n}$ and a $C^{1}$ function $w: S^{n} \rightarrow \mathbf{R}$ such that $\beta$ and $w$ are of class $C^{\infty}$ on $S^{n} \backslash\{a\}, \beta_{a}=\alpha_{a}$, $w_{a}=v_{a}, w>0$ on $S^{n}$ and for each $x$ in $\Sigma(f \circ \tau), \beta_{x}=\alpha_{x}$ and $w_{x}=v_{x}$. Set $g_{i}=w\left(f_{i} \circ \beta\right)$ for $i=1,2$. Clearly, $g_{i}$ is a $C^{\infty}$ function on $S^{n}$ and $g_{i}=\varphi_{i}$ on a neighborhood of $\Sigma(f \circ \tau) \cup\{\alpha\}$. Notice that the ideal $I\left(g_{1}, g_{2}\right)^{2}=I\left(\varphi_{1}, \varphi_{2}\right)^{2}$ is closed in $\mathscr{E}\left(S^{n}\right)$. Indeed, the ideal $I\left(\varphi_{2}, \varphi_{2}\right)^{2}$ can be generated by polynomial functions (choose the appropriate vector fields on $S^{n}$ ) and, hence, is closed [7]. Fix a polynomial function $\lambda$ in $I\left(g_{1}, g_{2}\right)^{2}$ with $\lambda^{-1}(0)=S\left(g_{1}, g_{2}\right)$. For each $i=1,2$, we can find a $C^{\infty}$ function $h_{i}: S^{n} \rightarrow \mathbf{R}$ satisfies

$$
g_{i}-\varphi_{i}=h_{i} \lambda \text { and } h_{i}(a)=0
$$

Let $\mu_{i}$ be a polynomial approximation to $h_{i}$ with $\mu_{i}(a)=0$. By Lemma 2.1 (with $Z=\{a\})$, there exist a $C^{\infty}$ diffeomorphism $\gamma: S^{n} \rightarrow S^{n}$ and a $C^{\infty}$ function $u: S^{n} \rightarrow \mathbf{R}$ such that $u>0, \gamma(a)=a$ and $\left(\psi_{1}, \psi_{2}\right)=\left(u\left(g_{1} \circ \gamma\right), u\left(g_{2} \circ \gamma\right)\right)$, where $\psi_{i}=\varphi_{i}+\mu_{i} \lambda$ for $i=1,2$. It follows that $f \circ \tau \circ \beta \circ \gamma \circ \rho^{-1}=\left(\psi_{1} / \psi_{2}\right) \circ \rho^{-1}$ on $\mathbf{R}^{n}$, where $\rho: S^{n} \backslash\{a\} \rightarrow \mathbf{R}^{n}$ is the stereographic projection. Notice that $\tau \circ \boldsymbol{\beta} \circ \gamma \circ \rho^{-1}$ is a $C^{\infty}$ diffeomorphism and $\left(\psi_{1} / \psi_{2}\right) \circ \rho^{-1}$ is a regular function. By [4], Theorem $8.4, f$ and $\left(\psi_{1} / \psi_{2}\right) \circ \rho^{-1}$ are analytically equivalent.

\section{References}

[1] J. Bochnak, W. Kucharz and M. Shiota, 'On equivalence of ideals of real global analytic functions and the 17th Hilbert problem', Invent. Math. 63 (1981), 403-421.

[2] J. Bochnak, W. Kucharz, and M. Shiota, 'On algebraicity of global real analytic sets and functions', Invent. Math. 70 (1982), 115-156.

[3] S. Lojasiewicz, Ensembles semi-analytiques (I.H.E.S., Lecture notes 1965).

[4] M. Shiota, 'Equivalence of differentiable mappings and analytic mappings', Inst. Hautes Etudes Sci. Publ. Math. 54 (1981), 37-122. 
[5] M. Shiota, 'Equivalence of differentiable functions, rational functions and polynomials', $A n n$. Inst. Fourier (Grenoble) 32 (1982), 167-204.

[6] R. Thom, 'L'équivalence d'une fonction différentiable et d'un polynôme', Topology 3, Suppl. 2 (1965), 297-307.

[7] J. Cl. Tougeron, Idéaux de fonctions différentiables, (Springer, Berlin-Heidelberg-New York. 1972).

Department of Mathematics

University of New Mexico

Albuquerque, New Mexico 87131

U.S.A. 